\title{
Monolithic thin film lithium niobate electro-optic modulator with over 110 GHz bandwidth
}

\author{
Fan Yang (杨 帆 $)^{1,2}$ ，Xiansong Fang (方先松) $)^{1,2}$ ，Xinyu Chen (陈心羽 $)^{1,2}$ ， Lixin Zhu (朱立新) ${ }^{1}$, Fan Zhang (张 帆) ${ }^{1,2}$ ， \\ Zhangyuan Chen (陈章渊) $)^{1,2}$, and Yanping Li (李艳萍) $)^{1,2^{*}}$ \\ ${ }^{1}$ State Key Laboratory of Advanced Optical Communication Systems and Networks, Department of Electronics, Peking University, Beijing 100871, China \\ ${ }^{2}$ Frontiers Science Center for Nano-optoelectronics, Peking University, Beijing 100871, China
}

*Corresponding author: liyp@pku.edu.cn

Received September 11, 2021 | Accepted November 2, 2021 | Posted Online November 24, 2021

\begin{abstract}
High-performance thin film lithium niobate (LN) electro-optic modulators with low cost are in demand. Based on photolithography and wet etching, we experimentally demonstrate a thin film LN Mach-Zehnder modulator with a $3 \mathrm{~dB}$ bandwidth exceeding $110 \mathrm{GHz}$, which shows the potential of boosting the throughput and reducing cost. The fabricated modulator also exhibits a comparable low half-wave voltage-length product of $\sim 2.37 \mathrm{~V} \cdot \mathrm{cm}$, a high extinction ratio of $>23 \mathrm{~dB}$, and the propagation loss of optical waveguides of $\sim 0.2 \mathrm{~dB} / \mathrm{cm}$. Besides, six-level pulse amplitude modulation up to $250 \mathrm{~Gb} / \mathrm{s}$ is successfully achieved.
\end{abstract}

Keywords: lithium niobate; electro-optic modulator; high bandwidth; photolithography; wet etching.

DOI: 10.3788/COL202220.022502

\section{Introduction}

Exponentially increasing global network traffic poses severe challenges to the bandwidth of optical transceivers. Electro-optic modulators (EOMs) with a large bandwidth, low-power consumption, a small footprint, and the possibility of large-scale manufacturing are in demand. In the past few years, tremendous efforts have been made towards a variety of platforms such as silicon $(\mathrm{Si})^{[1,2]}$, germanium-Si $(\mathrm{GeSi})^{[3]}$, indium phosphide $(\mathrm{InP})^{[4]}$, polymers ${ }^{[5]}$, plasmonics ${ }^{[6]}$, and thin film lithium niobate (TFLN $)^{[7-12]}$. Among them, monolithic TFLN EOMs, with etched lithium niobate (LN) waveguides, have attracted more and more attention since they retain excellent material and electro-optic (EO) properties while improving the ability of integration. Several monolithically integrated TFLN MachZehnder modulators (MZMs) ${ }^{[13-16]}$ demonstrated high bandwidth and low half-wave voltage-length product $\left(V_{\pi} L\right)$, which met the requirements for future photonic systems. In those high-performance devices, electron-beam lithography (EBL) with high-exposure resolution served a crucial function of defining high-quality waveguides or electrodes. However, the devices are still on the chip scale, need more exposure time, and are expensive for large-scale manufacturing. For application scenarios like data-center interconnects, EOMs with low cost are necessary. Therefore, photolithography is expected to be applied in the fabrication of monolithic TFLN EOMs due to its highefficiency exposure on the wafer scale. Previously, photolithography was utilized cooperating with dry etching, but the difficulty of obtaining smooth etching ${ }^{[17,18]}$ via dry etching limited the performance of the manufactured devices. A twostep masking technique ${ }^{[17]}$ and wet etching ${ }^{[18]}$ were proposed to address this problem. Recently, photolithography-exposed $\mathrm{EOMs}^{[19-21]}$ were demonstrated on the wafer scale. Although a low $V_{\pi} L^{[21]}$ was reported, further improvement of bandwidth is also a primary requirement for many applications. Figure 1 shows the comparison of the TFLN EOMs fabricated by EBL and photolithography.

In this paper, we present a TFLN EOM over $110 \mathrm{GHz}$ fabricated by wafer-scale ultraviolet (UV) photolithography and wet etching. Wet etching eliminates the influence of micro-masks and redeposition in dry etching methods, thereby obtaining high-quality waveguides and electrodes. Meanwhile, the half-wave $V_{\pi} L$ is $2.37 \mathrm{~V} \cdot \mathrm{cm}$, the extinction ratio is $>23 \mathrm{~dB}$, and the propagation loss of optical waveguides is $\sim 0.2 \mathrm{~dB} / \mathrm{cm}$, which indicate the overall superior performance. Based on our device, we also achieve modulation rates up to $250 \mathrm{~Gb} / \mathrm{s}$ of six-level pulse amplitude modulation (PAM-6) signals and $200 \mathrm{~Gb} / \mathrm{s}$ of PAM-4 signals. Our work demonstrates the feasibility of large-scale and low-cost manufacturing of LN EOMs and serves as an important step for making LN EOMs widely used in future cost-sensitive scenarios such as telecommunications and microwave photonics.

\section{Device Design and Fabrication}

The microscope image of the broadband LN modulator is shown in Fig. 2. The devices are fabricated on a commercial 4 in. ( 1 in. = $2.54 \mathrm{~cm}$ ) $x$-cut TFLN wafer (from NANOLN) with a $600 \mathrm{~nm}$ 


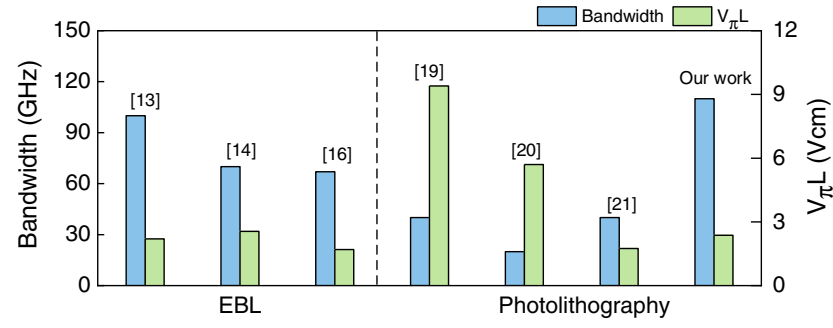

Fig. 1. Comparison of monolithic TFLN EOMs fabricated by EBL and photolithography.

thick LN layer, a $4.7 \mu \mathrm{m}$ thick thermal oxidation $\mathrm{SiO}_{2}$ layer, and a $525 \mu \mathrm{m}$ thick Si substrate. The modulator is working at the Mach-Zehnder interferometer configuration that consists of two $5 \mathrm{~mm}$ long modulation arms connected by $1 \times 2$ multimode interference (MMI) couplers to achieve beam splitting and beam combining. In order to realize efficient EO interaction, the electrodes are placed beside the TE mode ridge waveguides to ensure that the modal polarization and the electric field are both parallel to the direction of the largest EO coefficient $\gamma_{33}$ ( $z$ axis) in LN. In this configuration, the modulation signals are applied to the central electrode, while the outer electrodes are grounded. The modulation electric fields appearing in the two arms point in opposite directions, resulting in a push-pull operation. The electrodes are constructed by active regions and non-active regions. Active regions are made of coplanar waveguide (CPW) transmission lines, which can carry microwave signals propagating along the same direction as the optical wave. Non-active regions are set up for signal injection and termination. Two groups of ground-signal-ground (GSG) pads are erected and connected with the active regions through electrode tapers.

As is well known, there are three key factors for broadband operation of a traveling-wave modulator: (1) group-velocity matching between the optical wave in the waveguide and the microwave in the transmission line; (2) frequency-dependent attenuation of the microwave modulation signal as it propagates along the transmission line; (3) impedance matching between the microwave transmission line and external electrical connectors. Therefore, the bandwidth performance of the device is closely related to the electrode structure [width of central electrode $(W)$, gap between the electrodes $(G)$, electrodes thickness $(T)]$ and the dielectric layer, which is reflected in parameters of microwave loss $(\alpha)$, effective index $\left(N_{m}\right)$, and characteristic impedance $\left(Z_{0}\right)$. As displayed in Figs. 3(a)-3(c), $\alpha$ and $Z_{0}$ will both decrease with the increase of $W$, whereas $N_{m}$ goes up.
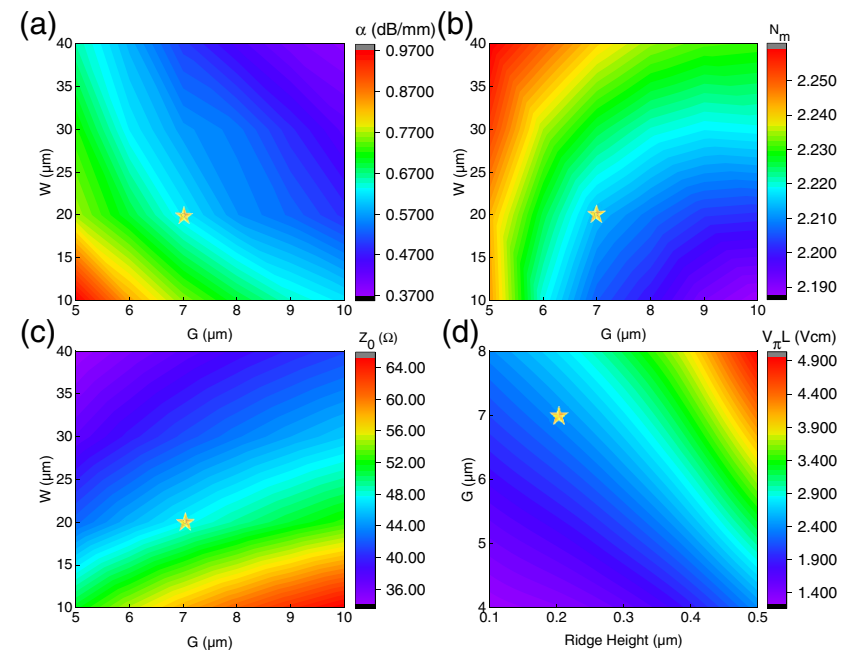

Fig. 3. Simulated results of (a) the microwave loss $(\alpha)$, (b) the effective index $\left(N_{m}\right)$ of microwave signals, and (c) the characteristic impedance $\left(Z_{0}\right)$ at $100 \mathrm{GHz}$ versus $W$ and $G_{;}(d)$ simulated $V_{\pi} L$ versus ridge height and $G$.

With the increase of $G, Z_{0}$ increases, and $N_{m}$ decreases, respectively. The $\alpha$ will also decrease when widening $G$ due to the alleviation of electrical current congestion in the gap. Besides, thickening the electrodes will bring down $N_{m}$ and $Z_{0}$ due to the increasing area of the air capacitor formed between the electrodes. However, it should be noticed that the expanded $G$ will cause the degeneration of the EO efficiency, which should be a design trade-off. Considering reducing $\alpha$ and the alignment tolerance of UV photolithography, we choose a slightly larger $G$ of $7 \mu \mathrm{m}$, while cooperating with shallow etched ridges of $200 \mathrm{~nm}$ to maintain a comparable $V_{\pi} L$ of $2.37 \mathrm{~V} \cdot \mathrm{cm}$, as shown in Fig. 3(d). $W$ and $T$ are jointly optimized to be, respectively, $20 \mu \mathrm{m}$ and $830 \mathrm{~nm}$ to ensure impedance matching and low $\alpha$. Since the waveguide is $1.3 \mu \mathrm{m}$ wide with $\mathrm{SiO}_{2}$ cladding, the effective optical group index $\left(N_{o}\right)$ is $\sim 2.24$ for the fundamental TE mode at $1550 \mathrm{~nm}$ simulated in Lumerical Mode Solution. Thanks to the low permittivity of the $\mathrm{SiO}_{2}(\varepsilon \sim 3.9)$ cladding and the $\mathrm{Si}$ $(\varepsilon \sim 11.9)$ substrate, the velocity matching design has an additional degree of freedom by adjusting the thickness of the cladding layer in addition to the electrode's design. The thickness of under-cladding and over-cladding is $4.7 \mu \mathrm{m}$ and $1 \mu \mathrm{m}$, respectively. Although the influence of the active regions dominates, the design of the non-active regions is also important at high frequency. We choose straight electrode tapers $(100 \mu \mathrm{m}$ long) and let the optical waveguides bend avoid extra $\alpha$ caused by electrodes bending. To maintain the continuity of the impedance of

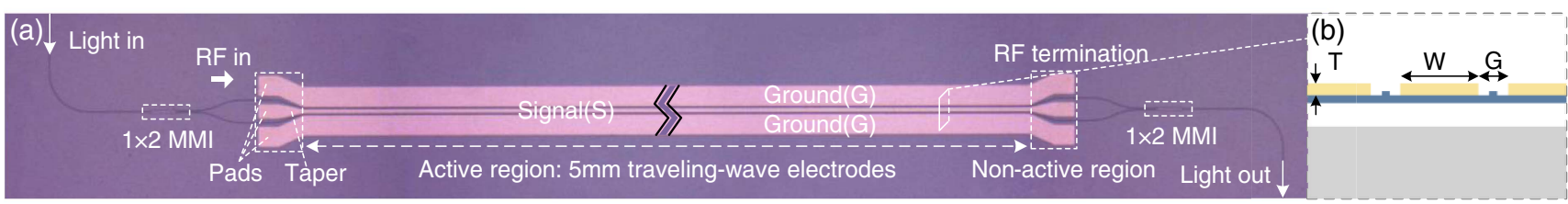

Fig. 2. (a) Microscope image and (b) cross section of the TFLN traveling-wave Mach-Zehnder EOM. 
the electrodes, the gap and width of the tapers are also designed to ensure that $Z_{0}$ equals $50 \Omega$.

Fabrication processes play an important role in the performance of final devices. Widely used dry etching methods with photoresists usually exhibit the problem of micro-masks, which will be transferred to the etched area causing roughness ${ }^{[17]}$. The roughness of the ridge induces high optical loss, while the roughness of the slab area affects the flatness of the electrodes placed there that induce high $\alpha$. To reduce the influence of roughness, we employ thin oxide masks and high-selectivity wet-etching. The TFLN MZM is fabricated as follows. It is worth noting that UV photolithography (by SUSS MA6) is used in all of the patterning. First, $100 \mathrm{~nm}$ thick $\mathrm{Si}$ oxide $\left(\mathrm{SiO}_{2}\right)$ is deposited on the LN wafer by plasma enhanced chemical vapor deposition (PECVD) as hard-masks in the following LN etching process. Then, the photoresist (AZ6112) patterns are transferred into the $\mathrm{SiO}_{2}$ layer through fluorine-based inductively coupled plasma dry etching. The power in the dry etching process is optimized at a low level to reduce roughness. After the removal of photoresist and etching residue, the circuits in the LN layer are produced by wet etching. Wet etching is developed in a mixed solution of $\mathrm{NH}_{4} \mathrm{OH}, \mathrm{H}_{2} \mathrm{O}_{2}$, and $\mathrm{H}_{2} \mathrm{O}$ with a ratio of 2:2:1 at $85^{\circ} \mathrm{C}$, which was used in the post-processing of LN waveguides ${ }^{[18]}$. The etching rate is $\sim 4 \mathrm{~nm} / \mathrm{min}$. During wet etching, the thickness of $\mathrm{SiO}_{2}$ masks is almost constant, which indicates a high selectivity. Finally, the $\mathrm{SiO}_{2}$ masks are removed with buffered oxide etching (BOE) solution. We have compensated for the width variation of the waveguides patterns in advance because wet etching leads to lateral etching. The original width of the waveguide is slightly larger than $1.3 \mu \mathrm{m}$, which has the advantage of reducing photolithography difficulty. CPW electrodes are formed using a standard lift-off process. After a thick negative photoresist is exposed, $50 \mathrm{~nm}$ of Ti is first deposited as an adhesive layer, and then $780 \mathrm{~nm} \mathrm{Au}$ is deposited with an electron-beam evaporator method. Afterwards, $1 \mu \mathrm{m}$ thick $\mathrm{SiO}_{2}$ cladding is deposited on top of all of the structures using PECVD. The $\mathrm{SiO}_{2}$ cladding on the pads is dry etched for probes contacts. The final devices are diced using the cleavage method to ensure smooth coupling faces. Hundreds of devices on a 4 in. wafer can be exposed in dozens of seconds by UV photolithography, yet it will take several days using EBL with a typical condition of $50 \mathrm{kV}$ acceleration voltage and $10 \mathrm{nA}$ beam current.

\section{Characterization and Transmission}

Figures 4(a) and 4(b) show the scanning electron microscope (SEM) micrographs of the device. The sidewall angle of the waveguide is nearly $90^{\circ}$ with respect to the horizonal direction. However, there is a $\sim 20^{\circ}$ slope at the bottom of one side, which is probably caused by the crystal orientation selectivity of wet etching. The root-mean-squared (RMS) roughness of the etched area is measured by atomic force microscopy (AFM), as shown in Fig. 4(c). In the slab area, the RMS roughness is about $0.3 \mathrm{~nm}$ over a $3 \mu \mathrm{m} \times 3 \mu \mathrm{m}$ area, which suggests there is little effect on the $\alpha$ of the electrodes placed on it. Extracted from the

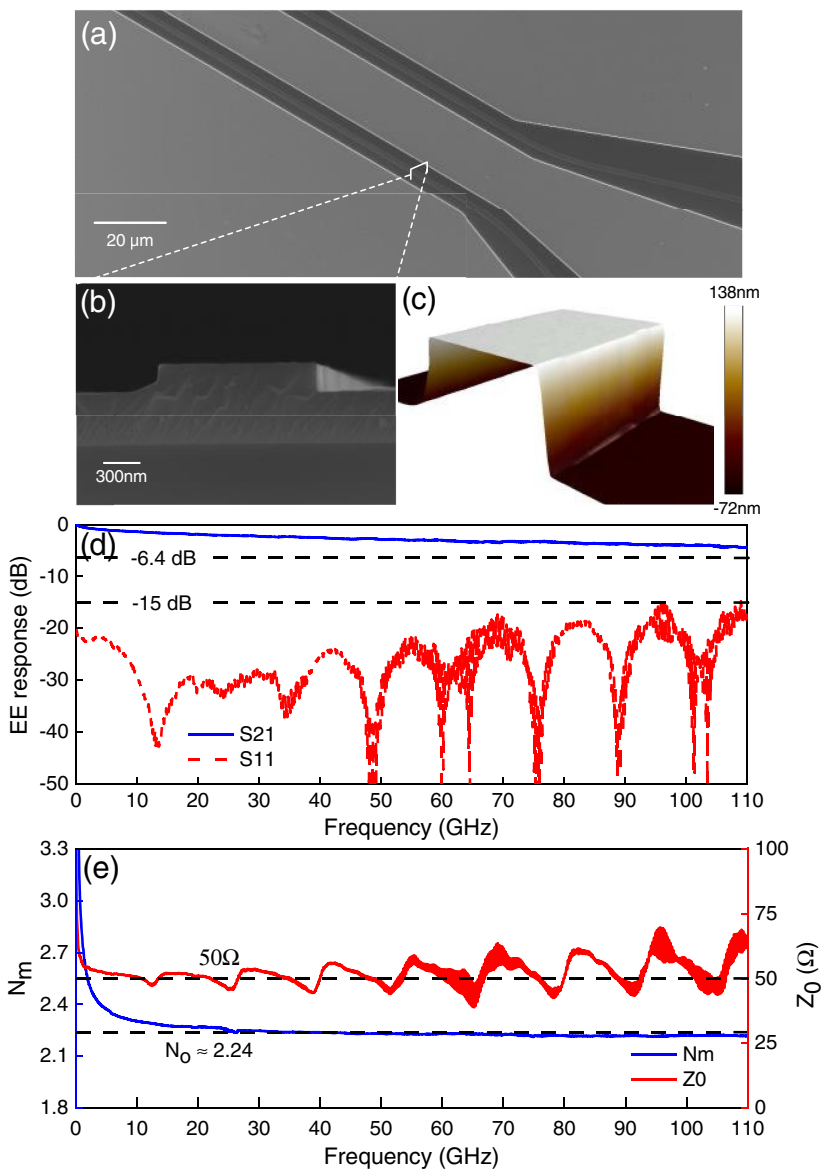

Fig. 4. (a) SEM image of the etched $\mathrm{LN}$ waveguides and electrodes before $\mathrm{SiO}_{2}$ cladding deposition. (b) SEM image of the cross section of the waveguides. (c) AFM measurement of the waveguides. (d) EE S-parameters of the CPW electrodes. (e) $Z_{0}$ and $N_{m}$ of the electrodes extracted from S-parameters in (d).

cutback method, the propagation loss of the $1.3 \mu \mathrm{m}$ wide waveguide is about $0.2 \mathrm{~dB} / \mathrm{cm}$. The total insertion loss of the device is $\sim 18 \mathrm{~dB}$, which is mainly from the mode mismatch between the lensed fiber and LN waveguide. Bending loss is also non-negligible. Optimizations of the coupling and bending waveguides are expected to produce a smaller total insertion loss. The most important part of the broadband modulator is the quality of the traveling-wave electrodes. In the case where the velocities of the optical wave and the microwave are perfectly matched, the $3 \mathrm{~dB}$ EO response can be estimated by the $6.4 \mathrm{~dB}$ bandwidth of the electrical-electrical (EE) transmission (S21). Hence, we characterize the CPW electrodes via high-frequency EE Sparameters, including S21 and reflection (S11) responses, as shown in Fig. 4(d). Two $110 \mathrm{GHz}$ GSG probes are used to launch and receive microwave power from the two ports of a Keysight N4372E vector network analyzer (VNA), respectively. The EE S21 curve shows a frequency response up to the measured $110 \mathrm{GHz}$ with a roll off of $4.5 \mathrm{~dB}$. We also extracted the $Z_{0}$ and the $N_{m}$ from the EE S-parameters in Fig. 4(e). The $Z_{0}$ is around $50 \Omega$ so that the reflection is weak, which can be seen from the S11 parameters maintaining below $-15 \mathrm{~dB}$ in Fig. $4(\mathrm{~d})$. 

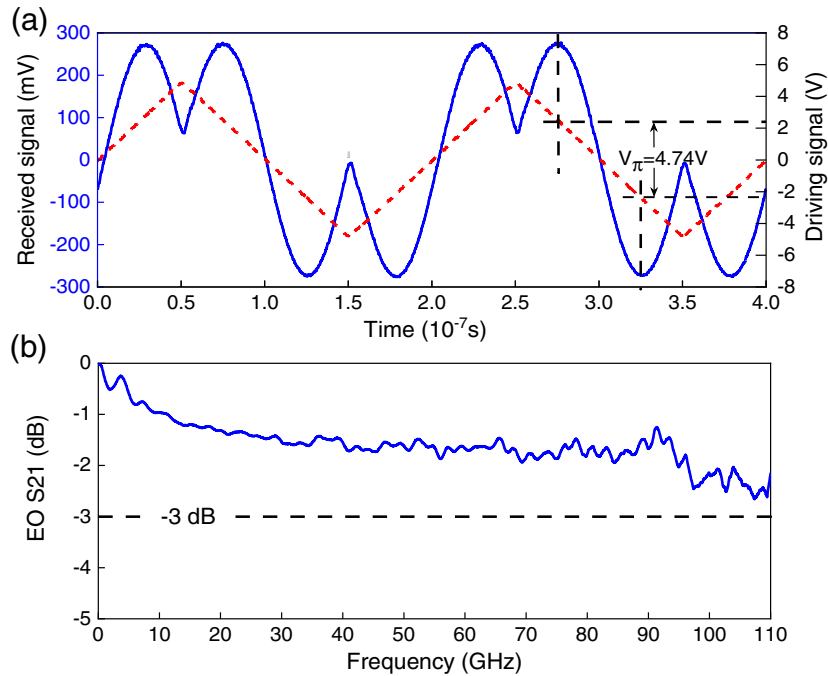

Fig. 5. EO characterization of the LN modulator: (a) $V_{\pi}$ test with $5 \mathrm{MHz}$ triangular voltages sweep. (b) EO S21 measurement result from $10 \mathrm{MHz}$ to $110 \mathrm{GHz}$, showing an ultra-high bandwidth over $110 \mathrm{GHz}$.

The $N_{m}$ is nearly equal to the group index of the optical mode $\left(N_{o}\right)$ with the difference below $2 \%$, which indicates nearly perfect velocity matching between the microwave and optical wave.

To verify the performance of the modulator, we first measure the $V_{\pi}$ with a triangular voltages sweep. We input a $5 \mathrm{MHz}$ triangular wave signal into the modulator and the oscilloscope at the same time. The modulated signal is received by a $1 \mathrm{GHz}$ photodiode (PD) to reduce the influence of out-of-band noise and subsequently loaded to the oscilloscope. The signals are shown

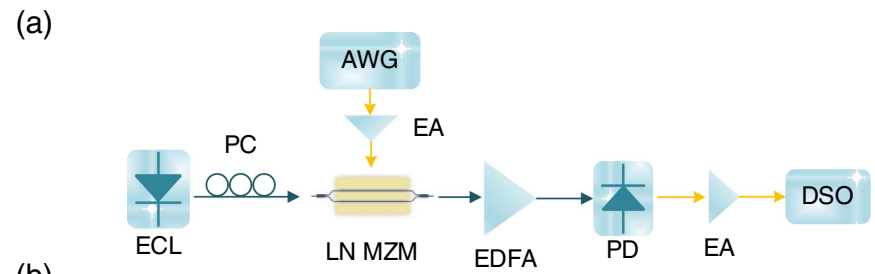

(b)

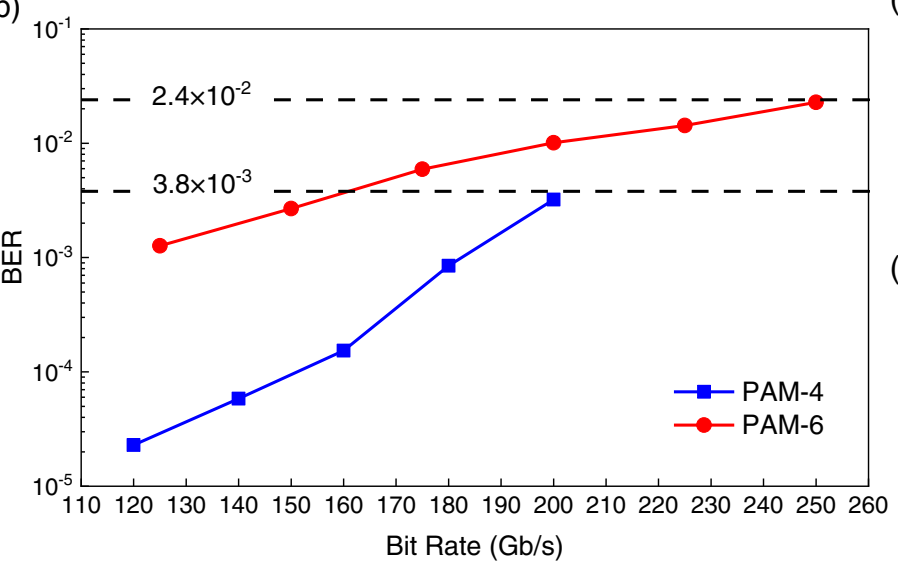

in Fig. 5(a). The measured $V_{\pi} L$ at $5 \mathrm{MHz}$ is $4.74 \mathrm{~V}$ for our $5 \mathrm{~mm}$ long device, which is comparable to the $V_{\pi} L$ in other references ${ }^{[13,14]}$. The extinction ratio of our modulator is $>23 \mathrm{~dB}$, which is obtained by DC measurement. Then, we show the broadband performance of our modulator through the smallsignal measurement of EO S21 [Fig. 5(b)]. The signals from the VNA are injected to the MZM with a $110 \mathrm{GHz}$ bias-T and a $110 \mathrm{GHz}$ probe. The CPW electrodes are terminated with an external $50 \Omega$ load through another $110 \mathrm{GHz}$ probe. Then, the received signals are sent to the VNA through the PD in the lightwave component analyzer (LCA). The EO response of the PD in the LCA is built-in, of which the influence will be automatically eliminated by the VNA during the test. The EO S21 presents a flat response over the frequency range with a roll-off of $\sim 2.5 \mathrm{~dB}$ from $10 \mathrm{MHz}$ to $110 \mathrm{GHz}$. Further optimizations of the highfrequency characteristics of the load and the electrodes structures are expected to improve the EO response further.

To evaluate the performance of our device, high-speed digital transmission exceeding $200 \mathrm{~Gb} / \mathrm{s}$ is performed. The experimental setup is depicted in Fig. 6(a). An external cavity laser at $1549.93 \mathrm{~nm}$ is employed as the optical source. The output laser power is $17 \mathrm{dBm}$. PAM-4/PAM-6 signals from an arbitrary waveform generator $(A W G)$ drive the modulator after being amplified by an electrical amplifier (EA). The modulated signals are amplified to $1 \mathrm{dBm}$ and subsequently detected by a singleended $70 \mathrm{GHz} \mathrm{PD}$, amplified by an EA, and sampled by a realtime digital storage oscilloscope (DSO) operating at $256 \mathrm{GSa} / \mathrm{s}$. Finally, the signals are processed by the receiver-side digital signal processing, which includes re-sampling, matched root raise cosine filter, synchronization, and Volterra non-linear equalization. Note that the frequency response of the whole system is

(c)

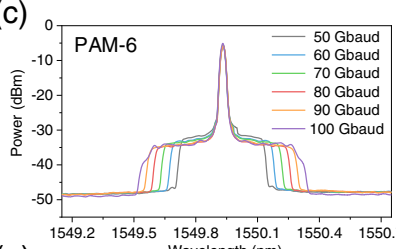

(e)
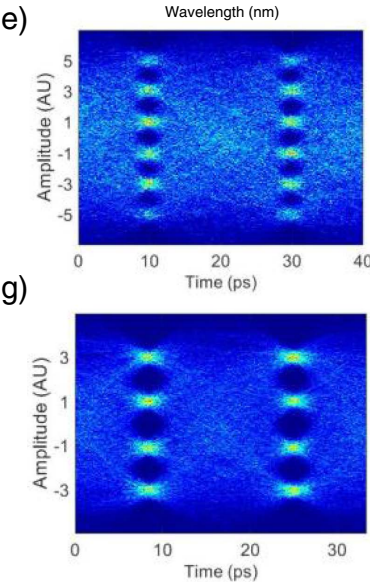

(d)

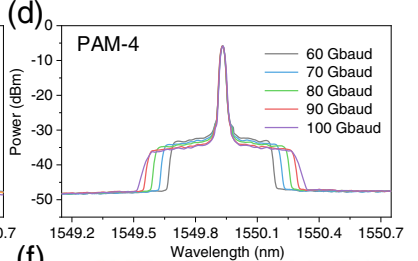

(f)
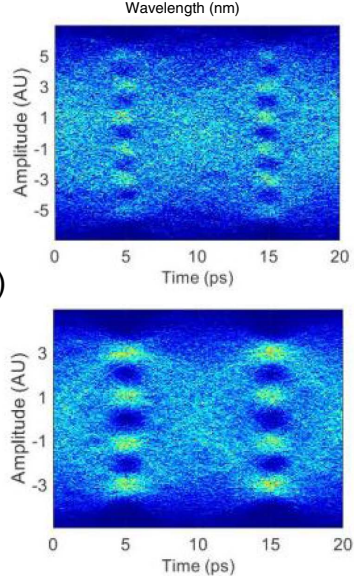

Fig. 6. Transmission results. (a) Experimental setup for the transmission system. (b) Measured BER performance versus bit rates of PAM-4 and PAM-6 signals. (c), (d) Optical spectrum of different baud rate PAM-4 and PAM-6 signals. (e)-(h) Typical eye diagrams of 50 Gbaud PAM-6, 100 Gbaud PAM-6, 60 Gbaud PAM-4, and 100 Gbaud PAM-4 signals, respectively. 
limited by typical components as $45 \mathrm{GHz}$ AWG and $50 \mathrm{GHz}$ EA instead of our modulator. Figures 6(c) and 6(d) show the measured optical spectrum of Nyquist PAM- 6 and PAM-4 signals in the back-to-back scenario, respectively. Figure 6(b) shows the measured bit error rates (BERs) of PAM- 6 and PAM-4 signals, respectively, for different data rates. The $250 \mathrm{~Gb} / \mathrm{s}$ PAM-6 signals and $200 \mathrm{~Gb} / \mathrm{s}$ PAM- 4 signals can be transmitted with BERs of $2.3 \times 10^{-2}$ and $3.2 \times 10^{-3}$, which are both below the $20 \%$ soft-decision forward error-correction (SD-FEC) threshold of $2.4 \times 10^{-2}$. Figures $6(\mathrm{e})-6(\mathrm{~h})$ show the typical eye diagrams of the received PAM- 6 and PAM- 4 signals. We believe that our device will achieve better BER performance if there is no additional electrical bandwidth limitation in the transmission system.

\section{Conclusion}

In this work, a TFLN MZM with a $3 \mathrm{~dB}$ bandwidth over $110 \mathrm{GHz}$ is experimentally demonstrated. The half-wave $V_{\pi} L$ is $2.37 \mathrm{~V} \cdot \mathrm{cm}$, and the extinction ratio is $>23 \mathrm{~dB}$, supporting $250 \mathrm{~Gb} / \mathrm{s}$ PAM-6 data transmission. With photolithography and wet etching, high-quality waveguides and traveling-wave electrodes are fabricated, which shows the potential of boosting the throughput and reducing cost. Although the presented performance has already met the requirements of many applications, tremendous efforts should also be made towards further improving the etching quality by combining dry etching and wet etching, reducing the coupling loss by designing the coupler structure, and so on. We believe that our work lays the groundwork for future applications of TFLN EOMs in cost-effective scenarios.

\section{Acknowledgement}

This work was supported by the National Natural Science Foundation of China (Nos. 61690194 and 61911530162). The authors acknowledge Jiyuan Wu, Haiquan Cheng, Haichuan Yang, and Cheng Zhang at Keysight's open laboratory for their help in the S-parameters measurement.

\section{References}

1. M. Li, L. Wang, X. Li, X. Xiao, and S. Yu, "Silicon intensity Mach-Zehnder modulator for single lane $100 \mathrm{~Gb} / \mathrm{s}$ applications," Photon. Res. 6, 109 (2018).

2. Y. Zhu, F. Zhang, F. Yang, L. Zhang, X. Ruan, Y. Li, and Z. Chen, "Toward single lane $200 \mathrm{G}$ optical interconnects with silicon photonic modulator," J. Lightwave Technol. 38, 67 (2020).

3. E. El-Fiky, P. De Heyn, M. Osman, A. Srinivasan, A. Samani, M. Pantouvaki, M. Sowailem, J. Van Campenhout, and D. Plant, "112 Gb/s PAM4 transmission over $2 \mathrm{~km}$ SMF using a C-band GeSi electro-absorption modulator," in Optical Fiber Communication Conference (Optical Society of America, 2018), paper W2A.16.
4. Y. Ogiso, J. Ozaki, Y. Ueda, N. Kashio, N. Kikuchi, E. Yamada, H. Tanobe, S. Kanazawa, H. Yamazaki, Y. Ohiso, T. Fujii, and M. Kohtoku, "Over $67 \mathrm{GHz}$ bandwidth and $1.5 \mathrm{~V} \mathrm{~V}_{\pi}$ InP-based optical IQ modulator with n-i-p-n heterostructure," J. Lightwave Technol. 35, 1450 (2017).

5. S. Wolf, H. Zwickel, C. Kieninger, Y. Kutuvantavida, M. Lauermann, J. Lutz, L. Altenhain, R. Schmid, W. Freude, C. Koos, and S. Randel, "Silicon-organic hybrid (SOH) IQ modulator for 100 GBd 16QAM operation," in Optical Fiber Communication Conference (Optical Society of America, 2017), paper Th5C.1.

6. C. Haffner, D. Chelladurai, Y. Fedoryshyn, A. Josten, B. Baeuerle, W. Heni, T. Watanabe, T. Cui, B. Cheng, S. Saha, D. L. Elder, L. Dalton, A. Boltasseva, V. M. Shalaev, N. Kinsey, and J. Leuthold, "Low-loss plasmon-assisted electro-optic modulator," Nature 556, 483 (2018).

7. L. Cai, Y. Wang, and H. Hu, "Low-loss waveguides in a single-crystal lithium niobate thin film," Opt. Lett. 40, 3013 (2015).

8. P. Weigel, J. Zhao, K. Fang, H. Al-Rubaye, D. Trotter, D. Hood, J. Mudrick, C. Dallo, A. Pomerene, A. Starbuck, C. DeRose, A. Lentine, G. Rebeiz, and S. Mookherjea, "Bonded thin film lithium niobate modulator on a silicon photonics platform exceeding $100 \mathrm{GHz} 3-\mathrm{dB}$ electrical modulation bandwidth," Opt. Express 26, 23728 (2018).

9. M. He, M. Xu, Y. Ren, J. Jian, Z. Ruan, Y. Xu, S. Gao, S. Sun, X. Wen, L. Zhou, L. Liu, C. Guo, H. Chen, S. Yu, L. Liu, and X. Cai, "High-performance hybrid silicon and lithium niobate Mach-Zehnder modulators for $100 \mathrm{Gbit} \mathrm{s}^{-1}$ and beyond," Nat. Photonics 13, 359 (2019).

10. M. Xu, M. He, Y. Zhu, L. Liu, L. Chen, S. Yu, and X. Cai, "Integrated thin film lithium niobate Fabry-Perot modulator [Invited]," Chin. Opt. Lett. 19, 060003 (2021).

11. Z. Wang, C. Wu, Z. Fang, M. Wang, J. Lin, R. Wu, J. Zhang, J. Yu, M. Wu, W. Chu, T. Lu, G. Chen, and Y. Cheng, "High-quality-factor optical microresonators fabricated on lithium niobate thin film with an electro-optical tuning range spanning over one free spectral range [Invited]," Chin. Opt. Lett. 19, 060002 (2021).

12. K. Zhang, Z. Chen, H. Feng, W. Wong, E. Y.-B. Pun, and C. Wang, "High-Q lithium niobate microring resonators using lift-off metallic masks [Invited]," Chin. Opt. Lett. 19, 060010 (2021).

13. C. Wang, M. Zhang, X. Chen, M. Bertrand, A. Shams-Ansari, S. Chandrasekhar, P. Winzer, and M. Loncar, "Integrated lithium niobate electro-optic modulators operating at CMOS-compatible voltages," Nature 562, 101 (2018).

14. M. Xu, M. He, H. Zhang, J. Jian, Y. Pan, X. Liu, L. Chen, X. Meng, H. Chen, Z. Li, X. Xiao, S. Yu, S. Yu, and X. Cai, "High-performance coherent optical modulators based on thin-film lithium niobate platform," Nat. Commun. 11, 3911 (2020).

15. P. Kharel, C. Reimer, K. Luke, L. He, and M. Zhang, "Breaking voltagebandwidth limits in integrated lithium niobate modulators using microstructured electrodes," Optica 8, 357-363 (2021).

16. X. Liu, B. Xiong, C. Sun, J. Wang, Z. Hao, L. Wang, Y. Han, H. Li, J. Yu, and Y. Luo, "Wideband thin-film lithium niobate modulator with low halfwave-voltage length product," Chin. Opt. Lett. 19, 060016 (2021).

17. K. Luke, P. Kharel, C. Reimer, L. He, M. Loncar, and M. Zhang, "Wafer-scale low-loss lithium niobate photonic integrated circuits," Opt. Express 28, 24452 (2020).

18. G. Ulliac, V. Calero, A. Ndao, F. I. Baida, and M.-P. Bernal, “Argon plasma inductively coupled plasma reactive ion etching study for smooth sidewall thin film lithium niobate waveguide application," Opt. Mat. 53, 1 (2016).

19. A. J. Mercante, P. Yao, S. Shi, G. Schneider, J. Murakowski, and D. W. Prather, "110 GHz CMOS compatible thin film $\mathrm{LiNbO}_{3}$ modulator on silicon," Opt. Express 24, 15590 (2016).

20. A. Mercante, S. Shi, P. Yao, L. Xie, R. Weikle, and D. Prather, "Thin film lithium niobate electro-optic modulator with terahertz operating bandwidth," Opt. Express 26, 14810 (2018).

21. Y. Liu, H. Li, J. Liu, S. Tan, Q. Lu, and W. Guo, "Low $\mathrm{V}_{\pi}$ thin-film lithium niobate modulator fabricated with photolithography," Opt. Express 29, 6320 (2021). 